\title{
USO DE NDVI PARA ANÁLISE DO COMPORTAMENTO DO REGIME DE VAZÕES DO RIO PARAOPEBA, MG
}

Use of NDVI for trend analysis of stream flow in the Paraopeba river, $M G$

\author{
Uilson Ricardo Venâncio Aires ${ }^{1 *}$; José de Oliveira Melo Neto² Jasmine Alves Campos ${ }^{3}$
}

\begin{abstract}
${ }^{1}$ Mestrando em Engenharia Agrícola; Departamento de Engenharia Agrícola; Universidade Federal de Viçosa; uvaires@gmail. ${ }^{2}$ Doutor em Recursos Hídricos em Sistemas Agrícolas; Instituto de Ciência e Tecnologia, Campus Poços de Caldas; Universidade Federal de Alfenas; joseneto_86@hotmail.com.

${ }^{3}$ Mestranda em Engenharia Agrícola; Departamento de Engenharia Agrícola; Universidade Federal de Viçosa; jasminealvescampos@gmail.com.
\end{abstract}

Artigo enviado em 28/04/2017, aceito em 06/07/2017 e publicado em 29/09/2017.

Resumo: O comportamento não estacionário de séries de dados hidrológicos configura-se como um desafio para a gestão e planejamento dos recursos hídricos, pois este modelo atual considera que não há alterações significativas nas séries hidrológicas ao longo do tempo. Neste contexto, o objetivo deste trabalho foi verificar o comportamento no regime fluviométrico no Rio Paraopeba, MG, em decorrência das modificações da cobertura vegetal. A estação fluviométrica utilizada está localizada no município de Juatuba, MG. A série possui período de observação de 76 anos (1938 a 2014). Para verificar a hipótese de estacionariedade e de tendência temporal foram utilizados os testes de Spearman e Mann Kendall com nível de significância de 5\%. Para a verificação da alteração da cobertura vegetal foi utilizado o Índice Vegetativo por Diferença Normalizada (NDVI), obtido através da plataforma Google Earth Engine (GEE) para os períodos: anual (janeiro a dezembro), chuvoso (outubro a março) e seco (abril a setembro). Observouse aumento das áreas de cobertura vegetal com alto vigor vegetativo na região correspondente a área de contribuição da estação fluviométrica, o qual pode ter influenciado na tendência de redução das vazões máximas e mínimas.

Palavras-chave: Testes não paramétricos, tendência temporal, Google Earth Engine

Abstract: The non-stationary behavior of hydrological records is a challenge for water resource's management and planning, once this current model considers that there are no significant changes in the hydrological records over the time. In this context, this study aims to verify the patterns of Paraopeba's river regime, MG, due the alterations in the land cover. It was used a 76-years flow records (1938 to 2014) from a fluviometric gauge located at Juatuba, MG. Spearman and Mann Kendall tests were tested in order to verify the hypothesis of stationarity and time trend at a $5 \%$ level of significance. For the verification of the land cover change was done using the Normalized Difference Vegetative Index (NDVI) obtained through the Google Earth Engine (GEE) platform for three seasonal periods: annual (January to December), rainy (October to March) and dry (April to September. The data demonstrated an increasing in land cover areas with high vegetative vigor in the region corresponding to the contribution area of the fluviometric station over the years, which may have affected both maximum and minimum flows trend.

Keywords: Non - parametric tests, temporal trend, Google Earth Engine

\section{INTRODUÇÃO}

As alterações climáticas apresentam impactos notáveis sobre o ciclo hidrológico, em especial, na modificação da frequência e amplitude da ocorrência de eventos hidrológicos extremos (ISHAK et al., 2013). Tal condição, configura-se como um problema para o sistema de gerenciamento dos recursos hídricos, pois estes são operados e projetados com base na hipótese de estacionariedade, ou seja, a ideia de que os sistemas naturais flutuam dentro de um ciclo imutável de variabilidade (MILLY et al., 2008), ou seja, não há ocorrência de mudanças significativas na série de dados ao longo do tempo (VILLARINI et al., 2011).

As mudanças nos padrões meteorológicos e seus efeitos no clima nas últimas décadas afetam significativamente a estacionariedade das séries de dados (ISHAK et al., 2013). Diversos estudos têm demostrado 
comportamento não estacionário da precipitação em diversas partes do globo, onde além da identificação de aumento dos eventos extremos, também foram evidenciadas redução na precipitação média anual (ISHIDA et al., 2017; SALVIANO et al., 2016; LI et al., 2016; WESTRA; SISSON, 2011).

No entanto, o regime de vazões apresenta maior sensibilidade, pois é influenciado, além das alterações climáticas (VILLARINI et al., 2011), por ações antrópicas nas bacias hidrográficas, como obras de infraestrutura, modificações nos canais do rio, drenagem e, em especial, as alterações na cobertura vegetal (MILLY et al., 2008).

As mudanças no regime de vazões são frequentemente observadas em um grande número de rios em todo o mundo como respostas as alterações ambientais (GAO et al., 2012). A redução da cobertura vegetal resulta em aumento nas vazões máximas e mínimas, devido principalmente a redução da infiltração da água no solo, favorecendo o escoamento superficial e a redução no processo de evapotranspiração (BOSCH; HEWLETT, 1982; SAHIN; HALL, 1996; COSTA et al., 2003; FARLEY et al., 2005; COE et al., 2011; KHANNA et al., 2017).

A atividade agropecuária no Brasil tem sido o fator com maior contribuição para a alteração na cobertura vegetal, com destaque para as regiões ocupadas com áreas de cerrado e vegetação nativa da região amazônica, provocando diversas mudanças ambientais (SALLO et al., 2014; COE et al., 2011; COSTA et al., 2003). Por conseguinte, analisar estas modificações em escala temporal e espacial é fundamental para a preservação dos recursos naturais, sendo a aplicação de técnicas de sensoriamento remoto de grande auxílio neste monitoramento, pois fornece informações em larga escala, e permite identificar em tempo real as alterações na cobertura vegetal através da análise de componentes agrometeorológicos, como albedo, índice de vegetação e evapotranspiração (GU et al., 2017; EDMONDS et al., 2016; SALLO et al., 2014; GOMES et al., 2009). Em seu estudo Ohana-Levi et al. (2015), visando modelar os efeitos da dinâmica da cobertura vegetal em bacias hidrográficas sobre $\mathrm{O}$ escoamento superficial, obtiveram uma relação de $93 \%$ $\left(\mathrm{R}^{2}\right)$ entre a modificação temporal dos valores de Índice de Vegetação por Diferença Normalizada (NDVI) com o escoamento superficial.

A plataforma de processamento de dados Google Earth Engine (GEE), lançada em 2013, se configura como uma ferramenta com grande potencial para auxiliar na obtenção de informações de sensoriamento remoto. Dentre os diversos produtos, esta plataforma online disponibiliza coleções de imagens
Landsat a partir de 1972 para qualquer região do globo e permite o processamento de dados em nuvem, o que reduz consideravelmente o tempo de processamento e de armazenamento de dados. Diversos trabalhos foram desenvolvidos utilizando esta plataforma, com destaque para mudanças na cobertura vegetal utilizando o NDVI (GU et al., 2017; EDMONDS et al., 2016; HUNTINGTON et al., 20016; KELLEY, et al., 2016; WARREN et al., 2015).

Compreender as características das mudanças no regime fluviométrico e analisar as razões destas mudanças é fundamental para uma gestão eficiente dos recursos hídricos (GAO et al., 2012). Neste contexto, o objetivo deste trabalho foi identificar possíveis alterações no regime fluviométrico no Rio Paraopeba, MG, e analisar a sua relação com as mudanças na vegetação na Bacia Hidrográfica do Rio Paraopeba (BHRP) através do NDVI obtido com o processamento de imagens Landsat na plataforma Google Earth Engine.

A erosão acelerada do solo tem sido largamente reconhecida como um problema ambiental no que tange à ocupação do solo para práticas agropecuárias e florestais, afetando sua capacidade produtiva, redução da porosidade do solo bem como sua capacidade de retenção e infiltração de água, com consequente aumento do escoamento superficial, do transporte de sedimentos e assoreamento de corpos hídricos.

Dessa forma, mudanças na cobertura do solo, biomassa e do regime hidrológico em bacias hidrográficas tende a ocorrer, afetando o processo erosivo, como sugerem Lee e Lee (2010) e Mello et al. (2015), podendo provocar alterações na morfologia fluvial (GUERRERO et al., 2013).

Segundo Pandey, Chowdary e Mal (2007), para um adequado manejo da bacia hidrográfica, visando à sustentabilidade dos recursos naturais, é necessário que se tenha informações espacializadas sobre o potencial erosivo dos solos e a produção e o transporte de sedimentos. Contudo, modelar o processo erosivo do solo é uma tarefa complexa em função das diversas interações dos fatores ativos e passivos que influenciam este processo.

Todavia, a avaliação quantitativa da erosão pode contribuir para a elaboração de possíveis estratégias para a gestão de bacias hidrográficas no contexto do desenvolvimento sustentável. Assim, modelos de simulação de erosão, especialmente modelos distribuídos, são úteis para avaliar diferentes estratégias 
de uso do solo e melhorar o seu manejo nessas bacias hidrográficas (BESKOW et al., 2009)

\section{MATERIAL E MÉTODOS}

O estudo foi realizado na área de contribuição da estação fluviométrica Ponte Nova do Paraopeba (código: 40800001), localizada na Bacia Hidrográfica do Rio Paraopeba (BHRP) da Unidade de Planejamento e Gestão dos Recursos Hídricos (UPGRH) Alto São Francisco.

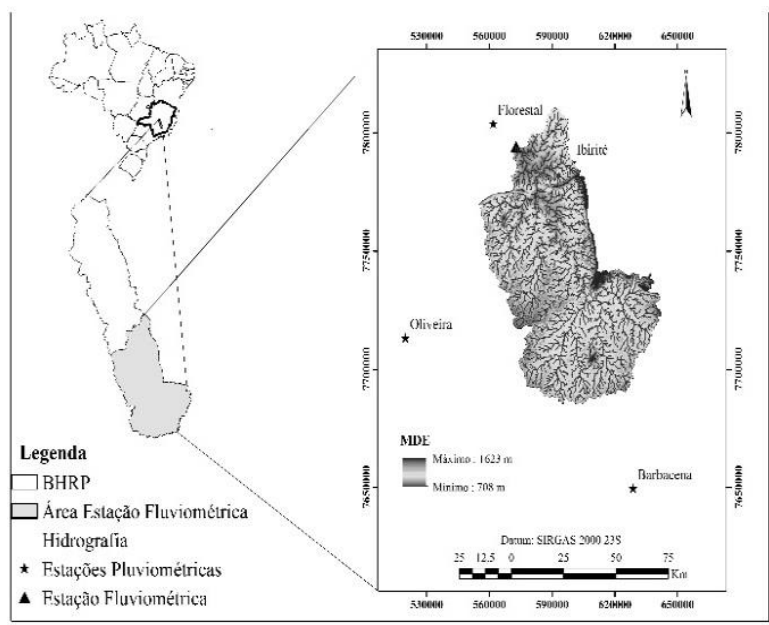

Figura 1 - Localização da área de contribuição da estação fluviométrica utilizada neste estudo
A estação fluviométrica Ponte Nova do Paraopeba pertence à rede de monitoramento da Agência Nacional de Águas (ANA) e está localizada no município de Juatuba, MG, com área de contribuição de $5.672,51 \mathrm{~km}^{2}$. A série de vazões foi obtida junto à plataforma HIDROWEB (2016) e possui período de observação de 76 anos (1938 a 2014). Foi realizada uma análise comparativa da variação das vazões entre os conjuntos de dados (1938 a 1984) e (1984 a 2014), para verificar o comportamento das vazões a partir de 1984 , pois a partir deste ano, há disponibilidade das imagens de NDVI, o que possibilita associar as mudanças na cobertura vegetal com o regime de vazões.

Foi verificado também o regime pluviométrico sobre a área de influência da estação fluviométrica, o qual poderia influenciar no regime de vazões, para tanto, utilizouse estações pluviométricas do Instituto Nacional de Meteorologia (INMET, 2016). Estas estações foram identificadas aplicando-se o método do Polígono de Thiessen. A localização das estações em coordenadas geográficas, os municípios em que estão instaladas e sua área de influência sobre BHRP podem ser verificados na Tabela 1.

Tabela 1 - Localização das estações meteorológicas do INMET que atuam sobre a BHRP

\begin{tabular}{|c|c|c|c|c|c|}
\hline Município & Longitude & Latitude & Altitude (m) & $\begin{array}{c}\text { Área de } \\
\text { influência }\left(\mathrm{km}^{2}\right)\end{array}$ & $\begin{array}{c}\text { Área de } \\
\text { influência (\%) }\end{array}$ \\
\hline Barbacena & $-43,78$ & $-21,24$ & 1126 & 1344,84 & 23,7 \\
\hline Florestal & $-44,42$ & $-19,89$ & 749 & 277,49 & 4,9 \\
\hline Ibirité & $-44,01$ & $-20,03$ & 815 & 3505,05 & 61,8 \\
\hline Oliveira & $-44,82$ & $-21,68$ & 967 & 545,13 & 9,6 \\
\hline
\end{tabular}

Fonte: INMET (2016)

As séries de precipitação contam com 55 anos de observações (1960 a 2015), com exceção da estação de Oliveira, com 70 anos de observações entre 1940 a 2010.

\section{Testes não paramétricos utilizados}

Foram aplicados os testes não paramétricos de Spearman e Mann Kendall para verificação do comportamento da vazão na bacia do rio Paraopeba, considerando-se o nível de significância de 5\%. No teste de Spearman, a hipótese $H_{0}$ afirma que os dados não apresentam tendência temporal, ou seja, a série é estacionária (MELLO; SILVA, 2013). A estatística do teste é dada pela Equação 1.

$$
T=\frac{c s}{\sqrt{\operatorname{Var}(c s)}}
$$


2 e 3.

O coeficiente ${ }^{c s}$ é calculado através das Equações

$$
\begin{aligned}
& c s=1-\frac{6 \sum_{i=1}^{N}\left(F_{i}-f_{i}\right)^{2}}{N^{3}-N} \\
& \operatorname{Var}(c s)=\frac{1}{N-1}
\end{aligned}
$$

$\mathrm{Na}$ Equação 2, $f_{i}$ é a posição temporal da observação na série histórica e $F_{i_{i}}$ é a posição que as observações, associadas a $f_{i}$ ocupam, com a série organizada em ordem crescente e $\mathrm{N}$ representa o tamanho da amostra de dados. A hipótese $\mathrm{H}_{0}$ pode ser rejeitada se $\left\{|T|>Z_{(\alpha / 2)}\right\}$.

O Teste de Mann Kendall é frequentemente empregado para identificar tendências em séries de dados climáticos, bem como para avaliar a ocorrência de mudanças climáticas (ZHONG et al., 2017; DEGEFU; BEWKET, 2017; GU et al., 2017). Neste teste, a variável S, para uma série de ${ }^{n}$ dados é calculada a partir da somatória dos sinais $(S g n)$ da diferença, par a par, de todos os valores da série $\left(x_{i}\right)$ em relação aos valores que a eles são futuros $\left(x_{j}\right)$, expressos nas equações 4 e 5 (SALVIANO et al., 2016).

$$
\begin{aligned}
& S=\sum_{i=1}^{n-1} \sum_{j=i+1}^{n} \operatorname{sgn}\left(x_{j}-x_{i}\right) \\
& \operatorname{Sgn}\left(x_{j}-x_{i}\right)=\left\{\begin{array}{l}
+1 ; \text { se } x_{j}>x_{i} \\
0 ; \text { se } x_{j}=x_{i} \\
-1 ; \text { se } x_{j}<x_{i}
\end{array}\right.
\end{aligned}
$$

Quando $n \geq 10$, a variável $S$ pode ser comparada com uma distribuição normal, sendo que a variância $(\operatorname{Var}(S))$, pode ser expressa pela equação 6 .

$\operatorname{Var}(S)=n \frac{(n-1)(2 n+5)-\sum_{i=1}^{n} t_{i}(i-1)\left(2_{i}+5\right)}{18}$

Em que ${ }^{t_{i}}$ representa a quantidade de repetições de uma extensão $i$.

A tendência observada na série histórica é considerada crescente ou decrescente de acordo com os valores positivos e negativos do indicie $Z_{M K}$, respectivamente. De acordo com o sinal de $S$, o indicie $Z_{M K}$ é calculado a partir da equação 7 .

$$
\begin{aligned}
& Z_{M K}=\frac{S-1}{\sqrt{\operatorname{Var}(S)}} ; \operatorname{para} S>0 \\
& Z_{M K}=0 ; \operatorname{para} S=0 \\
& Z_{M K}=\frac{S-1}{\sqrt{\operatorname{Var}(S)}} ; \operatorname{para} S<0
\end{aligned}
$$

Rejeita-se a hipótese $H_{a}$, ou seja, a série apresenta tendência temporal, se o valor absoluto de $Z_{\mathrm{MK}}$ for superior ao valor tabelado $Z_{\alpha / 2}$.

\section{Obtenção dos dados de NDVI}

O NDVI tem sido um bom indicador das alterações na vegetação em decorrência, por exemplo, do desmatamento. Este índice apresenta variação de -1 a 1, em que áreas ocupadas por vegetação mais densa tendem a apresentar valores de NDVI próximos a 1, pois uma vegetação verde e sadia mostra evidente contraste entre a região do visível, especificamente no vermelho e infravermelho próximo e que, quanto maior for esse contraste, haverá maior vigor da vegetação na área imageada (ZHONG et al., 2017; MOREIRA, 2012). O NDVI pode ser obtido através da equação 8.

$N D V I=\frac{I V P+V e r}{I V P-V e r}$

Em que IVP é a energia refletida na região do infravermelho próximo; e $V e r$, a energia refletida na região do vermelho do espectro eletromagnético.

Realizou-se a verificação a dinâmica do NDVI em 30 anos na área de drenagem da estação fluviométrica, com base nos seguintes anos: 1984 e 1994, em que se utilizou imagens de Landsat 5. Verificou-se também os valores de NDVI para ano de 2004, sendo que para este ano empregou-se imagens do Landsat 7, e para 2014, utilizandose imagens do Landsat 8 . Foi feita também a verificação de ganho e perda de área de cobertura vegetal entre 1984 e 2014. As imagens utilizadas possuem resolução espacial de 30 metros e já apresentam correções radiométrica e atmosférica.

O processamento dos dados de NDVI foi realizado na plataforma online do Google Earth Engine 
(https://code.earthengine.google.com/), que trabalha com linguagem de programação JavaScript. No estudo de Hansen et al. (2013), em que realizou um mapeamento global das áreas de florestas e suas perdas e ganhos, em termos de área, utilizou-se 20 terapixels de dados de Landsat processados nesta plataforma. O processamento de dados foi completado em questão de dias, o qual levaria 15 anos se utilizasse um único computador (LEE et al., 2016). Os dados de entrada para as análises estão disponíveis na própria interface da plataforma GEE, sendo necessário formular os Scripts para a obtenção das informações de interesse que estão conectadas a diversos servidores, como exemplo, o Earth Explore.

As imagens de NDVI apresentam os valores médios por pixel referentes ao período anual (janeiro a dezembro), período seco (abril a setembro) e período chuvoso (outubro a março), pois através da plataforma GEE foi aplicado um algoritmo de remoção de nuvens utilizando a banda cfmask das imagens Landsat Surface Refectancia (SR), o que permite utilizar todas as imagens disponíveis em cada período e gerar uma única imagem de saída com valores médios.

Para análise da mudança temporal e espacial da mudança na cobertura vegetal classificou-se os valores de NDVI em 5 classes proposta por Borges (2010) para o Estado de Minas Gerais, conforme a Tabela 2.

Tabela 2 - Classificação dos valores de NDVI

\begin{aligned} & \hline NDVI \multicolumn{1}{c}{ Interpretação } \\ & \hline$-1,0-0,0$ Ausência de Cobertura Vegetal \\ & $0,0-0,2$ Vigor vegetativo baixo \\ & $0,2-0,4$ Vigor vegetativo moderado \\ & $0,4-0,6$ Vigor vegetativo alto \\ & $0,6-1,0$ Vigor vegetativo muito alto \\ & \hline\end{aligned}

Fonte: Borges (2010).

\section{RESULTADOS E DISCUSSÃO}

O regime pluvial da BHRP tem como característica dois períodos bem definidos, com o período seco concentrado entre os meses de abril a setembro e período chuvoso entre outubro a março. A Tabela 3 apresenta os valores de precipitação em termos médios para o período seco e chuvoso e precipitação anual para cada estação com área de influência na área de contribuição da estação fluviométrica utilizada, além dos valores associados à série sintética gerada pelo método dos Polígonos de Thiessen.

Tabela 3 - Precipitação em termos médios nas estações pluviométricas

\begin{tabular}{llll}
\hline Estação Pluviométrica & $\begin{array}{c}\text { Média } \\
\text { anual } \\
\text { (mm) }\end{array}$ & $\begin{array}{c}\text { Período } \\
\text { seco } \\
\text { (mm) }\end{array}$ & $\begin{array}{c}\text { Período } \\
\text { chuvoso } \\
\text { (mm) }\end{array}$ \\
\hline Oliveira & 1374 & 182 & 1191 \\
Ibirité & 1467 & 174 & 1293 \\
Florestal & 1387 & 151 & 1236 \\
Barbacena & 1398 & 219 & 1179 \\
Série sintética (Thiessen) & 1389 & 166 & 1223 \\
\hline
\end{tabular}

Em termos médios, observa-se que a estação de Ibirité registra os maiores valores de precipitação anual e também para o período chuvoso, enquanto que a estação de Barbacena apresenta o maior volume precipitado no período seco.

A Figura 2 apresenta os valores anuais das vazões máxima (a), mínima (b) e média (c) para o período de 1938 a 2014

$\mathrm{Na}$ Figura 2, pode-se observar uma tendência de redução nos regimes de vazão, com destaque para a vazão média e mínima. Apesar da vazão máxima aparentar comportamento estacionário ao longo do tempo, ainda pode-se notar uma leve tendência de redução, com uma taxa de redução média de $-7,46$ m³/s por ano entre 1938 a 2014 .

De maneira geral, observa-se redução nos regimes fluviométricos na análise comparativa. A vazão máxima apresentou a maior redução entre os meses de fevereiro e março 49,0\% e 60,8\%, respectivamente. A vazão mínima também apresentou as maiores reduções nestes meses, com $73,1 \%$ em fevereiro e 68,3\% em março. Já as vazões médias apresentaram maiores reduções no mês de outubro (20,3\%) e fevereiro $(17,4 \%)$. 

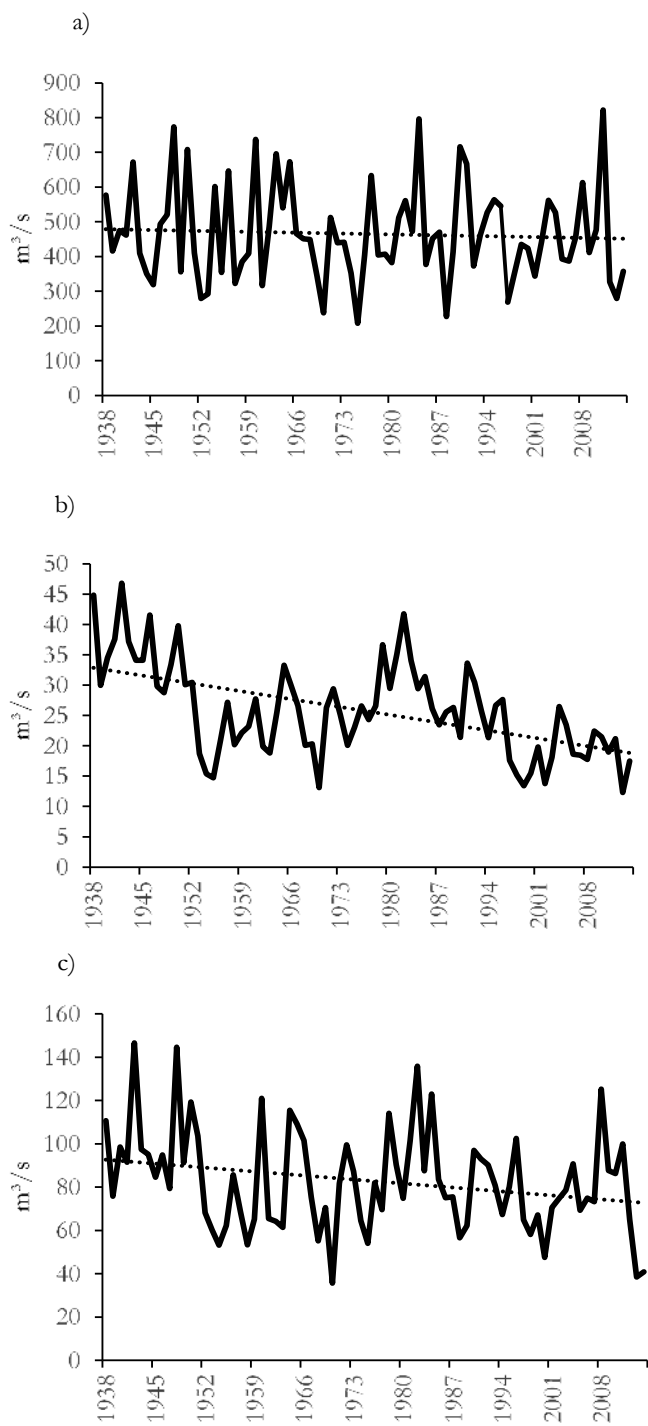

Figura 2 - Valores das vazões máxima (a), mínima (b) e média (c) entre 1938 a 2014

A Tabela 4 apresenta os valores mensais, em termos médios, para as vazões máxima, mínima e média das séries analisadas.
Tabela 4 - Valores médios das vazões máxima, mínima e média

\begin{tabular}{|c|c|c|c|}
\hline \multirow{2}{*}{ Mês } & \multicolumn{3}{|c|}{ Período: 1938 - 2014} \\
\hline & $Q \max \left(\mathrm{m}^{3} / \mathrm{s}\right)$ & $Q \min \left(\mathrm{m}^{3} / \mathrm{s}\right)$ & Qmed $\left(\mathrm{m}^{3} / \mathrm{s}\right)$ \\
\hline Jan & 282,86 & 54,8 & 162,78 \\
\hline $\mathrm{Fev}$ & 240 & 55,2 & 139,5 \\
\hline Mar & 185,03 & 53,51 & 125,64 \\
\hline Abr & 132,5 & 46,65 & 84,03 \\
\hline Mai & 85,2 & 39,26 & 60,81 \\
\hline Jun & 74,05 & 33,61 & 50,88 \\
\hline Jul & 61,82 & 29,86 & 42,96 \\
\hline Ago & 73,62 & 25,01 & 36,83 \\
\hline Set & 94,63 & 21,74 & 38,04 \\
\hline Out & 156,48 & 21,78 & 47,39 \\
\hline Nov & 247,73 & 26,7 & 74,72 \\
\hline Dez & 295,24 & 40,4 & 126,92 \\
\hline
\end{tabular}

Nota-se pela Tabela 4 que, em termos médios, a maior vazão máxima apresenta ocorrência no mês de dezembro. Enquanto que a menor vazão mínima é observada no mês de agosto. Já a vazão média apresenta o maior valor no mês de janeiro e menor valor com ocorrência no mês de agosto.

A Figura 3 apresenta a variação no regime de vazões, em termos médios entre os conjuntos de dados (1938 a 1984) e (1984 a 2014).

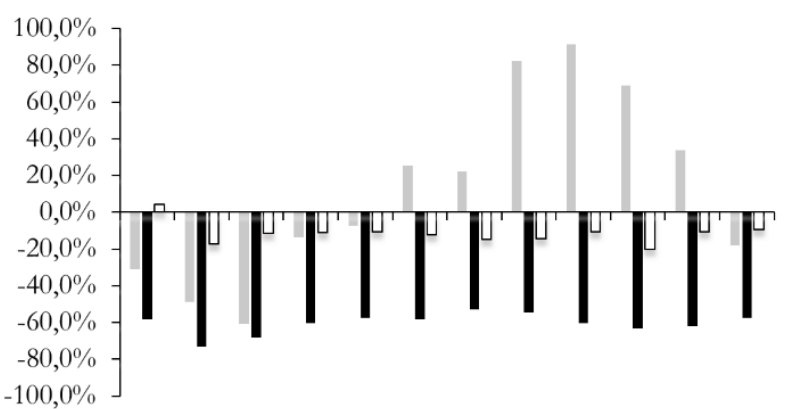

jan fev mar abr mai jun jul ago set out nov dez $\because \mathrm{Q} \max \backsim \mathrm{Qmin} \quad \square \mathrm{Qmed}$

Figura 3 - Variações no Regime de vazões em termos médios 
Os resultados obtidos com a aplicação dos Testes de Spearman e Mann Kendall, para identificar o comportamento da série de vazão da BHRP, encontram-se na Tabela 5.

Tabela 5 - Valores obtidos na aplicação dos Testes de Spearman e Mann Kendall

\begin{tabular}{llll}
\hline \multicolumn{5}{c}{ Coeficiente de Spearman } \\
\hline Índice & Qmax & Qmin & Qmed \\
Nível de significância $(\alpha)$ & 0,05 & 0,05 & 0,05 \\
$|\mathrm{~T}|$ & 3,027 & 7,009 & 4,505 \\
$\mathrm{Z}(\alpha)$ & 1,96 & 1,96 & 1,96 \\
\hline \multicolumn{5}{c}{ Mann Kendall } \\
\hline Índice & Qmax & Qmin & Qmed \\
Nível de significância $(\alpha)$ & 0,05 & 0,05 & 0,05 \\
$Z_{(}(\alpha)$ & 1,96 & 1,96 & 1,96 \\
$Z_{\mathrm{MK}}$ & $-3,2$ & $-7,18$ & $-4,67$ \\
\hline
\end{tabular}

Com a aplicação do Teste de Spearman, constatouse que as séries de vazão apresentaram comportamento não estacionário. Com nível de significância de 5\%, obteve-se $|\mathrm{T}|$ de 3,027, 7,009 e 4,505 paras as vazões máximas, mínimas e médias, respectivamente. Com estes resultados, foi verificada a presença de tendência temporal nas séries.

O Teste de Mann Kendall também apontou tendência de alteração temporal em ambos os regimes de vazão do Rio Paraopeba. Como o valor absoluto de $Z_{M K}$ calculado foi superior ao nível de significância de $5 \%$, as séries apresentam tendência temporal de redução em ambos os regimes de vazão na BHRP.

A bacia do rio Paraopeba apresenta extensa demanda pelo uso da água (usos consultivos e insignificantes), de acordo com Durães (2013), considerando os usos insignificantes, na estação Ponte Nova do Paraopeba, observou-se uma demanda de água até 2013 de 14,52 m³ s. Já para 2014, foi verificado junto a Secretaria de Estado de Meio Ambiente e Desenvolvimento Sustentável (SEMAD, 2017), que para este ano específico foram deferidas outorgas para dois empreendimentos localizados na área de contribuição desta estação, totalizando um acréscimo na captação de água de $1,0 \mathrm{~m}^{3} / \mathrm{s}$. Em contra partida, a vazão máxima e média para o período entre 1938 e 2014 apresentaram uma redução de 169,5 m³ / s e $49,8 \mathrm{~m}^{3} / \mathrm{s}$, respectivamente, e para as vazões mínimas, uma redução de $9,0 \mathrm{~m}^{3} / \mathrm{s}$, o que representa $46,4 \%$ da vazão mínima média para este período. Desta forma, as variações nos regimes de vazão indicam que a redução observada pode estar relacionada com outros fatores além do uso da água outorgado na área de contribuição da estação fluviométrica.

Congo et al., (2009), analisaram as mudanças anuais do regime de vazões do Yellow River, e constataram que o aumento da demanda por água e a redução do regime pluviométrico foram os principais fatores que contribuíram com a seca do rio, especialmente no ano de 1990. Desta forma, é fundamental o entendimento do comportamento da precipitação sobre a bacia.

Os resultados obtidos referentes as tendências do regime pluviométrico sobre BHRP aplicando-se o Teste de Mann Kendall são apresentadas na Tabela 6.

Tabela 6 - Analise de tendência nas estações da BHRP

\begin{tabular}{lcc}
\hline \multirow{2}{*}{ Estações } & \multicolumn{2}{c}{ Parâmetros } \\
\cline { 2 - 3 } & $\mathbf{Z}_{\mathbf{M K}}$ & $\mathbf{Z}_{(\alpha)}$ \\
\hline Barbacena & 0,56 & 1,96 \\
Florestal & $-0,39$ & 1,96 \\
Ibirité & $-0,55$ & 1,96 \\
Oliveira & $-1,92$ & 1,96 \\
Série sintética (Thiessen) & $-0,17$ & 1,96 \\
\hline
\end{tabular}

A análise de tendência nas estações pluviométricas que têm influência sobre a área de drenagem da estação fluviométrica, demonstra que não ocorreu mudanças significativas no regime de chuvas. A estação de Oliveira, que apresentou o maior valor de $Z_{M K}$, o que pode estar associado ao período disponível de dados desta estação (1940 a 2010), com um maior número de dados observados, porém ainda é inferior ao nível de significância adotado.

\section{Alterações da cobertura vegetal na BHRP}

$\mathrm{Na}$ Figura 4 pode-se observar o NDVI médio em cada grau de vigor vegetativo para os períodos: anual, seco e chuvoso. 
a)

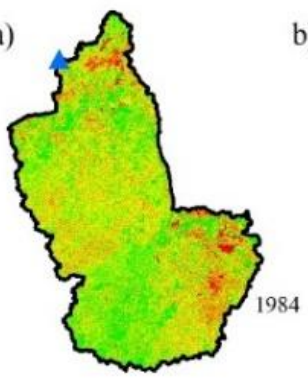

e)

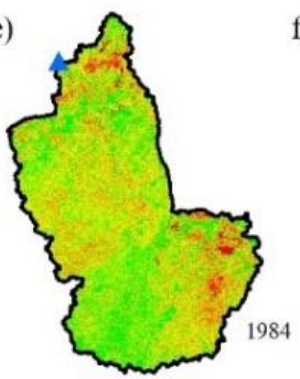

i)

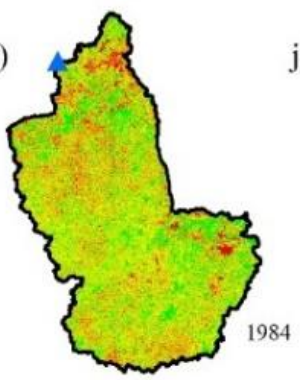

b)

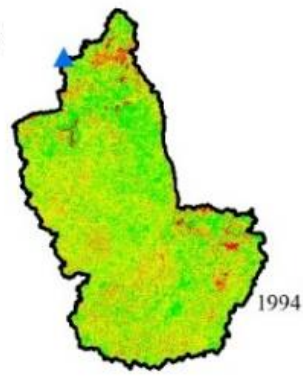

f)
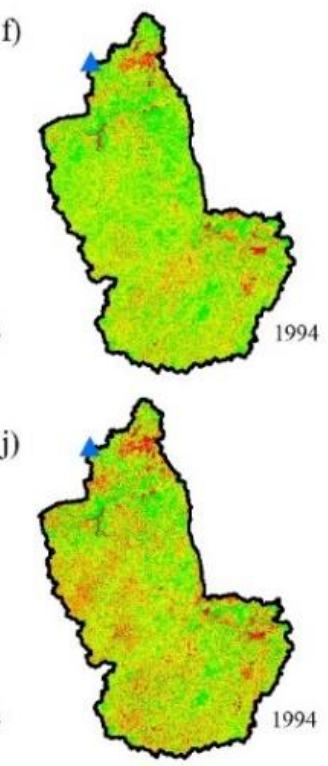

c)

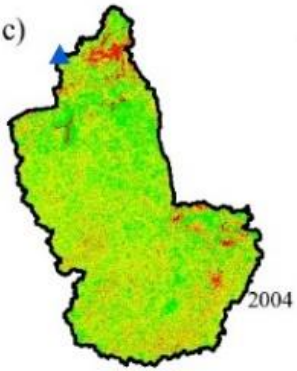

g)
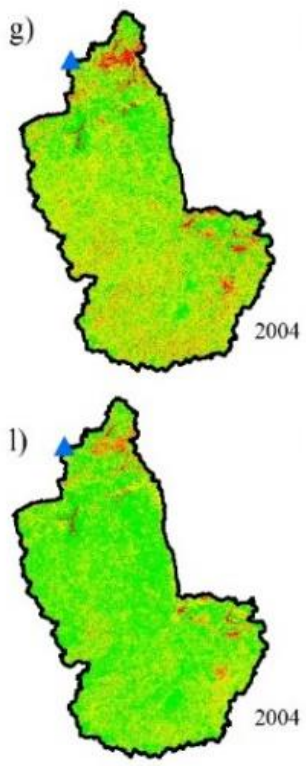

d)

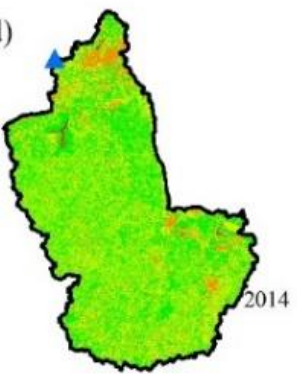

h)
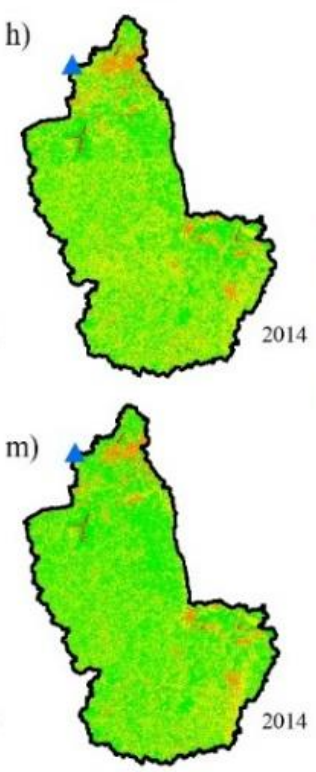

Legenda

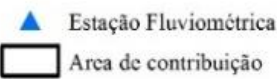

Grau de Vigor Vegetativo

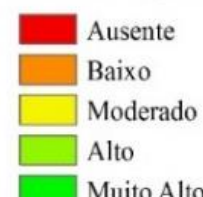

Muito Alto

Figura 4 - Mudança temporal e espacial do NDVI médio para os períodos anual (Figura 4(a) a Figura 4 (d)), seco (Figura 4 (e) a Figura 4 (h)) e chuvoso (Figura 4 (i) a Figura 4 (m)) entre os anos de 1984, 1994, 2004 e 2014 na área de contribuição da estação.

Nota-se na Figura 4 que houve um aumento nos valores de NDVI nas classes de alto e muito alto grau de vigor vegetativo, representando um ganho em cobertura vegetal no período de 1984 a 2014 na área de análise para os três períodos anual, seco e chuvoso. A Tabela 7 apresenta a estatística descritiva dos valores de NDVI na área de contribuição da estação fluviométrica.
Verifica-se pela Tabela 7 que ocorre um aumento nos valores médios e máximos de NDVI ao longo dos períodos de análise, o que reflete o aumento nas áreas com valores de alto e muito alto grau de vigor vegetativo identificados na Figura 4.

A Figura 5 apresenta os ganhos e perdas de área, em porcentagem, para cada classe de NDVI na região correspondente a área de contribuição da estação fluviométrica entre 1984 e 2014, para os períodos seco, chuvoso e anual. 
Tabela 7 - Estatística descritiva dos valores de NDVI na área de contribuição da estação fluviométrica NDVI período anual

\begin{tabular}{lrrrr} 
Estatística descritiva & $\mathbf{1 9 8 4}$ & $\mathbf{1 9 9 4}$ & $\mathbf{2 0 0 4}$ & $\mathbf{2 0 1 4}$ \\
\hline Mínimo & $-0,49$ & $-0,44$ & $-0,58$ & $-0,73$ \\
Máximo & 0,89 & 0,89 & 0,92 & 0,94 \\
Média & 0,5 & 0,56 & 0,59 & 0,63 \\
Desvio Padrão & 0,13 & 0,13 & 0,14 & 0,16 \\
\hline
\end{tabular}

NDVI período seco

Estatística descritiva $1984 \quad 1994 \quad 2004 \quad 2014$

\begin{tabular}{lrrrr}
\hline Mínimo & $-0,46$ & $-0,51$ & $-0,59$ & $-0,92$ \\
Máximo & 0,87 & 0,9 & 0,93 & 0,95 \\
Média & 0,51 & 0,56 & 0,59 & 0,61 \\
Desvio Padrão & 0,12 & 0,13 & 0,15 & 0,17 \\
\hline
\end{tabular}

NDVI período chuvoso

\begin{tabular}{lrrrr} 
Estatística descritiva & $\mathbf{1 9 8 4}$ & $\mathbf{1 9 9 4}$ & $\mathbf{2 0 0 4}$ & $\mathbf{2 0 1 4}$ \\
\hline Mínimo & $-0,49$ & $-0,51$ & $-0,47$ & $-0,92$ \\
Máximo & 0,84 & 0,9 & 0,91 & 0,93 \\
Média & 0,49 & 0,49 & 0,67 & 0,67 \\
Desvio Padrão & 0,12 & 0,15 & 0,13 & 0,17 \\
\hline
\end{tabular}

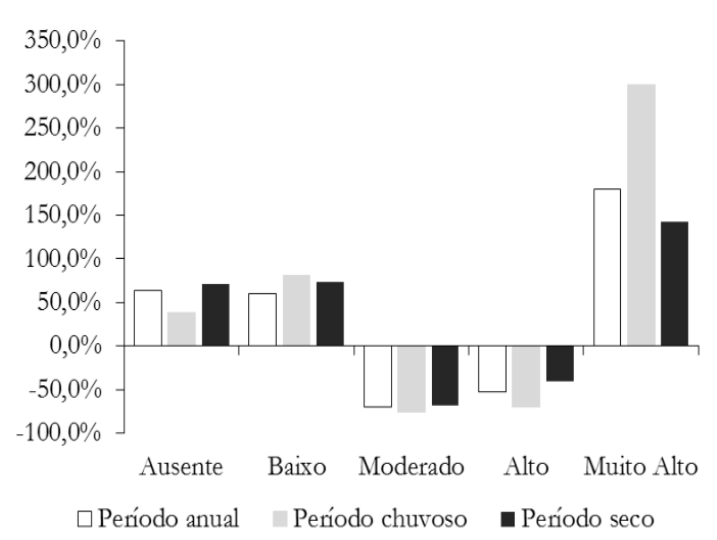

Figura 5 - Ganhos e perda de área em porcentagem

A Figura 5 evidencia que, na região correspondente a área de contribuição da estação fluviométrica, ocorreu um aumento de 179,8\%, 299,8\% e $142,5 \%$ de área correspondente a classe de NDVI com grau de vigor vegetativo muito alto para os períodos anual, chuvoso e seco. Este aumento corresponde a um ganho de $2.388 \mathrm{~km}^{2}$ de áreas com vigor vegetativo muito alto no período anual, $3.202 \mathrm{~km}^{2}$ para o período chuvoso e $1.956 \mathrm{~km}^{2}$ para o período seco.

$\mathrm{O}$ aumento na cobertura vegetal pode ter influenciado na tendência de redução observada no regime das vazões máxima, mínima e média ao longo do tempo. A redução na cobertura vegetal tende a resultar em um aumento no regime de vazões máximas, principalmente na vazão de pico, e nas vazões mínimas, pois apesar do aumento da taxa de infiltração também ocorre um incremento nos processos evapotranspirativos (COE et al., 2011; FARLEY et al., 2005; COSTA et al., 2003; SAHIN; HALL, 996; BOSCH; HEWLETT, 1982).

A Tabela 8 apresenta a análise de correlação entre a variação do regime de vazões com o aumento de área na classe de vigor vegetativo muito alto em cada período de análise.

Tabela 8 - Valores dos coeficientes de correlação $\left(\mathrm{r}^{2}\right)$

\begin{tabular}{cccc}
\hline & \multicolumn{3}{c}{ Coeficiente de determinação $\left(\mathbf{r}^{2}\right)$} \\
\cline { 2 - 4 } & $\begin{array}{c}\text { Período } \\
\text { seco }\end{array}$ & $\begin{array}{c}\text { Período } \\
\text { chuvoso }\end{array}$ & $\begin{array}{c}\text { Período } \\
\text { anual }\end{array}$ \\
\cline { 2 - 4 } Qmax & 0,09 & 0,41 & 0,16 \\
Qmin & 0,38 & 0,23 & 0,29 \\
Qmed & 0,21 & 0,71 & 0,27 \\
\hline
\end{tabular}

Nota-se pela Tabela 8 que o período chuvoso foi o que mais apresentou correlação com o regime de variação de vazões, em especial para as vazões máxima $(0,41)$ e média $(0,71)$. Já para a vazão mínima, a maior correlação foi alcançada no período seco $(0,38)$. No estudo de Latuf (2007), foi possível obter uma relação de $92 \%$ entre o regime de vazão média com a precipitação anual e a evolução da área de floresta como variáveis explicativas, e obteve também uma correlação de $44 \%$ entre o regime de vazão máxima com o mês mais chuvoso e a evolução da área de floresta. 


\section{CONCLUSÃO}

O regime de vazões no Rio Paraopeba, na seção de Ponte Nova, apresentou comportamento não estacionário ao longo do tempo com tendências de redução, como verificado através dos Testes de Mann Kendall e Spearman.

A alteração no regime de vazões pode estar relacionada com o aumento de áreas com alto vigor vegetativo, em que observou-se ganho de até $3.202 \mathrm{~km}^{2}$ para o período chuvoso $(56,4 \%$ da área de contribuição da estação fluviométrica) detectada pela análise temporal do NDVI.

\section{REFERÊNCIAS}

ANA. AGÊNCIA NACIONAL DE ÁGUAS.

Metadados. Disponível em:<

http://metadados.ana.gov.br/geonetwork/srv/pt/mai n.home?uuid $=\mathrm{e} 2 \mathrm{~d} 38 \mathrm{e} 3 \mathrm{f}-5 \mathrm{e} 62-41 \mathrm{ad}-87 \mathrm{ab}-$

990490841073 > acesso em: 24 jun. 2016.

HidroWeb: sistemas de informações hidrológicas.

Disponível em:

<http://hidroweb.ana.gov.br/HidroWeb>. Acesso em: 31 abr. 2016.

BORGES, L. A. Estimativa de NDVI no Estado de Minas Gerais utilizando imagens dos satélites CERBS_2BCDe MSG \SEVIRI. 2010. 60 p. Dissertação (Mestrado em Engenharia Agrícola) - Universidade Federal de Lavras, Lavras, 2010.

BOSCH, J. M.; HEWLETT, J. D. A review of catchment experiments to determine the effect of vegetation changes on water yield and evapotranspiration. Journal of Hydrology, v.85, p. 3-23, 1982.

CONG, Z.; YANG, D.; GAO, B.; YANG, H.; HU, H. Hydrological trend analysis in the Yellow River Basin using a distributed hydrological model. Water Resour. Res., v. 45, p. 1-13, 2009.
COE, M. T.; LATRUBESSE, E. M.; FERREIRA, M. E.; AMSLER, M. L. The effects of deforestation and climate variability on the streamflow of the Araguaia River, Brazil. Biogeochemistry, v. 105, p. 119-131, 2011.

COsTA, M. H.; BOTTAB, A.; CARDILLE, J. A. Effects of large-scale changes in land cover on the discharge of the Tocantins River, Southeastern Amazonia. Journal of Hydrology, v. 283, p. 206-2017, 2003.

DEGEFU, M. A.; BEWKET, W. Variability, trends, and teleconnections of stream flows with large-scale climate signals in the Omo-Ghibe River Basin, Ethiopia. Environ Monit Assess, v. 17, p. 189-142, 2017.

DURÃES, M. F. Desenvolvimento do indice de perturbação hidrossedimentológica: aplicação nas bacias hidrográficas dos rios Paraopeba e Sapucaí 224 p. Tese (Doutorado em Recursos Hídricos em Sistemas Agrícolas) Universidade Federal de Lavras, Lavras, 2013.

EDMONDS, D.; HAJEK, E. A.; DOWNTON, N.; BRYK, A. B. Avulsion flow-path selection on rivers in foreland basins. The Geological Society, v. 44, p. 695-698, 2016.

FRANCIS, J.; MAGILLIGAN, K. H. Nislow Changes in hydrologic regime by dams. Geomorphology, v.71, p. 61-78, 2005.

FARLEY, K. A.; JOBBÁGY, E. G.; JACKSON, R. B. Effects of afforestation on water yield: a global synthesis with implications for policy. Global Change Biology, v.11, p.1565-1576, 2005.

GAO, B.; YANG, D.; ZHAO, T.; YANG, H. Changes in the eco-flow metrics of the upper Yangtze river from 1961 to 2008. Journal of Hydrology, v. 448, p. 30-38, 2012.

GOMES, H. F.; SILVA, B. B.; CAVALCANTI, E. P.; ROCHA, H. R. Balanço de radiação em diferentes 
biomas no Estado de São Paulo mediante imagens Landsat 5. São Paulo, UNESP. Geociências, v. 28, n. 2, p. 153-164, 2009.

GU, X.; ZHANG, Q.; SINGH, V. P.; SHI, P. Changes in magnitude and frequency of heavy precipitation across China and its potential links to summer temperature. Journal of Hydrology, v. 547, p. 718-731, 2017.

HANSEM, M. C.; POTAPOV, P. V.; MOORE, R.; HANCHER, M.; TURUBANOVA, S. A.; TYUKAVINA, A.; THAU, D.; STEHAMAN, S. V.; GOETZ, S. J.; LOVELAND, T. R.; KOMMAREDDY, A.; EGROV, A.; CHINI, L.; JUSTICE, C. O.; TOWNSHEND, J. R. G. Highresolution global maps of 21 st-century forest cover change. Science, v. 342, p. 850-853, 2013.

HUNTINGTON, J.; MCGWIRE, K.; MORTON, C.; SNYDER, K.; PETERSON, S.; ERICKSON, T.; NISWONGER, R.; CARROLL, R.; SMITH, G.; ALLEN, R. Assessing the role of climate and resource management on groundwater dependent ecosystem changes in arid environments with the Landsat archive. Remote Sensing of Environment, v. 1851, p.86-197, 2016.

\section{INMET - INSTITUTO NACIONAL DE}

METEOROLOGIA. Séries históricas: dados mensais.

Disponível em: <

http://www.inmet.gov.br/projetos/rede/pesquisa/for m_mapas_mensal.php> acesso em: 22 jun. 2016.

ISHIDA, K.; GORGUNER, M.; ERCAN, A.; TRINH, T. KAVVAS, M. L. Trend analysis of watershed-scale precipitation over Northern California by means of dynamically-downscaled CMIP5 future climate projections. Science of the Total Environment, v. 592, p. $12-$ 24, 2017.

ISHAK, E. H.; RAHMAN, A.; WESTRA, S.; SHARMA, A.; KUCZERA, G. Evaluating the nonstationarity of Australian annual maximum flood. Journal of Hydrology, v. 494, p. 134-145, 2013.
LATUF, M. O. Mudanças no uso do solo e comportamento hidrológico nas bacias do rio Preto e Ribeirão Entre Ribeiros. 2007. 103 p. Dissertação (Mestrado em Engenharia Agrícola) - Universidade Federal de Viçosa, Viçosa, 2007.

LEE, J. S. H.; WICH, S.; WIDAYATI, A.; KOH, L. P. Detecting industrial oil palm plantations on Landsat imagens with Google Earth Engine. Remote Sensing Applications: Society and Environment, v.04, p. 219-224, 2016.

LI, J.; ZHU, Z.; DONG, W. Assessing the uncertainty of CESM-LE in simulating the trends of mean and extreme temperature and precipitation over China. International Journal of Climatology, v. 37, p. 2101 - 2110, 2016.

KELLEY, L. C.; EVANS, S. G.; POTTS, M. D. Richer histories for more relevant policies: 42 years of tree cover loss and gain in Southeast Sulawesi, Indonesia. Global Change Biology, v. 10, p. 1-10, 2016.

KHANNA, J.; NEDVIGY, D.; FUEGLISTALER, S.; WALKO, R. Regional dry-season climate changes due to three decades of Amazonian deforestation. Nature Climate Change, v. 07, p.200 -207, 2017.

MELLO, C. R.; SILVA, A. M. Hidrologia: Princípios e aplicações em sistemas agricolas. Lavras: UFLA, 2013. p. 163-247.

MOREIRA, M. A. Fundamentos do Sensoreamento Remoto e Metodologias de Aplicaşão. Viçosa, UFV, 2012. 422 p.

MILLY, P. C. D.; BETANCOURT, J.; FALKENMARK, M.; HIRSCH, R. M.; KUNDZEWICZ, Z. W.; LETTENMAIER, D. P.; STOUFFER, R. J. Stationarity Is Dead: Whither Water Management?. Science, v. 319, p. 573-574, 2008. 
OHANA-LEVI, N.; KARNIELI, A.; EGOZI, R.; GIVATI, A.; PEETERS, A.; Modeling the Effects of land-Cover Change on Rainfall-Runoff relationships in a Semiarid, Eastern Mediterranean Watershed. Advances in Meteorology, v. 10, p.1-16, 2015.

SAHIN, V., HALL, M. J., 1996. The effects of afforestation and deforestation on water yields. Journal of Hydrology, v. 178, p. 293-309, 1996.

SALVINO, N. F.; GROPPO, J. D.; PELLEGRINO, G. Q. Análise de Tendências em Dados de Precipitação e Temperatura no Brasil. Revista Brasileira de Meteorologia, v. 31, p. 64-73, 2016.

SALLO, F. S.; FRANÇA, M. S.; MORAIS, D. M.; RODRIGUES, R.; BIUDES, M. S. Estimativa de componentes do balanço de radiação em diferentes tipos de uso e cobertura do solo. Rev. Ambiente e Água, v. 9, p. 347-358, 2014.

SEMAD. SECRETARIA DO ESTADO DE MEIO AMBIENTE E DESENVOLVIMENTO

SUSTENTÁVEL. Outorgas: Relação deferidos, indeferidos, cancelados e outros. Disponível em: <

2011. http://www.meioambiente.mg.gov.br/outorga/relacao -deferidos-indeferidos-cancelados-e-outros $>$ acesso em: 02 jun. 2017.

VILLARINI, G.; SMITH, J. A.; SERINALDI, F.; NTELEKOS, A. A. Analyses of seasonal and annual maximum daily discharge records for central Europe. Journal of Hydrology, v. 399, p. 209-312, 2011.

ZHONG, K.; ZHENG, F.; WU, H.; QIN, C.; XU, X. Dynamic changes in temperature extremes and their association with atmospheric circulation patterns in the Songhua River Basin, China. Atmospheric Research, v. 190, p. 77-88, 2017.

WARREN, M. S.; BRUMBY, S. P.; SKILLMAN, S. W.; KELTON, T.; WOHLBERG, B.; MATHIS, M.; CHARTRAND, R.; KEISLER, R; JOHNSON, M. Seeing the Earth in the Cloud: Processing One Petabyte of Satellite Imagery in One Day. IEEE, v. 8, p. 1-12, 2015.

WESTRA, S.; SISSON, S. A. Detection of nonstationarity in precipitation extremes using a max-stable process model. Journal of Hydrology, v. 406, p. 119-128, 